\title{
Common Buckwheat-based EST Primers in the Genome of Other Species of Fagopyrum
}

\author{
Bal K. Joshi*, Kazutoshi Okuno, Ryo Ohsawa and Takashi Hara \\ Laboratory of Plant Breeding, Graduate School of Life and Environmental Sciences, University of \\ Tsukuba, Japan \\ * Current address: Nepal Agricultural Research Council, Kathmandu, Nepal \\ <joshibalak@yahoo.com>
}

\begin{abstract}
If the EST primers designed for one species can be used in related species, then the cost involved in developing markers for DNA fingerprinting, genetic relationship studies, mapping, etc. for other species is significantly reduced. We tested the applicability of 17 EST primers developed from common buckwheat in other wild and cultivated Fagopyrum species. A total of 18 accessions consisting of 4 subspecies and 2 species were used. Sequences of 93 cDNA clones were used to design primers using Primer3. Amplification products were different in band intensity. In most of the cases, the bands of $F$. homotropicum were with high intensity. All primers showed single band except in Accession C9022. Three primers 23, 31 and 69 produced very clear singe band. All primers amplified the genomic DNA of $F$. homotropicum (2x). Eight primers amplified the DNA of all accessions. Results indicated that the transferability of EST markers developed for common buckwheat decreased with an increase in genetic distance between them.
\end{abstract}

Key words: Common buckwheat, EST markers, Fagopyrum species, transferability

\section{INTRODUCTION}

Dominant molecular markers eg RAPD, AFLP, etc are expected to provide little or no information regarding synteny. Because EST PCR markers are derived from gene coding regions they are more likely to be conserved across populations and species than markers derived from random regions of DNA. EST is a partial sequence of a gene and is mapped on the respective gene position on the genome either by PCR based marker or RFLP probe. Mapping information can be compared among genomes in different species by the comparison of genetic sequences (Sato et al 2005).

The ability to transfer mapped STS markers within the Poaceae family has been demonstrated, both between cereal species (Erpelding et al 1996) and between cereal and forage grass species (Taylor et al 2001). STS primers developed from black spruce cDNA sequences have been shown to direct amplification in several different conifer species in the study of Rowland and Bhanaraj (2003) that tested whether many of the EST PCR primer pairs developed for blueberry are capable of amplifying DNA fragments in other members of the family Ericaeae. Of 26 primer pairs tested in cranberry 23 $(89 \%)$ resulted in successful amplification. Of 39 primer pairs tested in rhododendron $29(74 \%)$ resulted in successful amplification.

Applicability of SSR of Chamaecyparis obtusa has been assessed in 11 other species from five genera in the Cupressaceae and in Cryptomeria japonica (Nakao et al 2001). The evolutionary conservation of DNA sequences flanking mircrosatellite sites allows microsatellite markers developed in one species to be used in other related species (Echt et al 1999). Species within the same genera show similar tendencies for PCR amplification. The applicability test of the microsatellite markers developed in $C$. 
obtusa indicates that possible applications to other species are restricted even within the Cuppressaceae. The low applicability of $C$. obtusa microsatellite markers to other related species may be due to the genetic diversity of the species within the Cupressaceae (Nakao et al 2001).

Amplification was more consistent using the species-specific primer design for each gene (Yu et al 2004). Forty-four percent of the primers designed specifically for wheat sequences were successful in amplifying DNA from both wheat and rice species. EST-derived markers are likely to be more highly conserved, and therefore may be more transferable between species than anonymous sequence derived markers (Taylor et al 2001, Yu et al 2004). Moreover, ESTs that share homology to expressed genes can be specifically targeted for genetic mapping and can be useful for aligning genomes of distantly related species for comparative analysis. EST-derived SSRs have been evaluated on rice, durum wheat, barley and hexaploid wheat. In general, EST-SSRs have demonstrated less polymorphism compared to genomic SSRs, but have showed a high frequency of cross-species transferability. Yu et al (2004) tested EST-SSR primers developed from hexaploid wheat and rice ESTs on four cereal species (wheat, rice, barley, maize) and $62 \%$ of the functional primers produced PCR amplicons from two or more of the species. $44 \%$ of the primers derived from wheat EST sequences amplified rice DNAs. Goff et al (2002) reported that 59\% of rice cDNA sequences from Harushima et al (1998) matched two or more copies in the rice genome sequence identified by a BLASTN analysis. SSRs derived from ESTs are especially valuable as molecular markers because they are derived from gene transcripts and are more likely to be conserved among species (Yu et al 2004).

Although there are many advantages to using EST-PCR markers, considerable cost is involved in terms of time, money, and other resources. In developing this type of marker RNA must be extracted from sometimes small amounts of the appropriate tissue, a cDNA library must be constructed, hundreds of clones must be picked and plasmid DNA isolated from them, the clones must be sequenced, and primers must be designed, synthesized, and tested. If the EST-PCR primers designed for one species can be used in related species, then the cost involved in developing markers for DNA fingerprinting, genetic relationship studies, mapping, etc. for other species is significantly reduced. In addition, if the markers are shown to be homologous, they can be used for comparative mapping studies between the species. In general, the wider the taxonomic range across which primers are transferable, the more economically attractive primer development becomes. Successful amplification is only one measure of utility. Therefore we tested the applicability of EST primers developed from common buckwheat in other Fagopyrum species.

\section{MATERIALS AND METHODS}

\section{DNA extraction}

A total of 18 accessions consisting of 4 subspecies and 2 species (Table 1) were used. Seeds were collected from Kyoto University, Japan. These accessions were seeded in Growth Chamber of Plant Breeding Lab of Tsukuba University, Japan. Leaves from 22 day old seedlings were collected in plastic bag. Two leaves per accession, one leaf from each individual plant were collected. About 8 pieces were made from a single leaf and one piece was used to extract the DNA. Remaining leaf pieces were kept in $2 \mathrm{ml}$ size tube and stored in deep fridge. DNA was extracted from the fresh piece of each leaf using the kit method (DNeasy Plant Mini Kit). Two DNA samples from each accession were used in this study. The extracted DNA was stored in deep fridge and used directly without diluting for further study.

DNA was checked in 2\% Agarose $\mathrm{S}$ gel containing $2.5 \%$ ethidium bromide in $1 \times$ TAE buffer (Figure 1). Electrophoresis was run at $100 \mathrm{~V}$ for $30 \mathrm{~min}$. Gels were stained with ethidium bromide and visualized and documented using Kodak 1D software (Kodak EDAS290). Two DNA samples from each accession were loaded to insure DNA extraction. 


\section{Primers design}

Sequences of 384 cDNA clones provided by Dr J. Aii were used to design primers after removing primer sequences. These sequence data was used in Standard BLAST search to identify unique sequences. 93 clones had unique sequences and these clones were used to design primers. The primer was constructed in summary as follows: (1) identify the sequence of interest from the cDNA library clones; (2) insert the sequence into the input window of the Web-based PCR primer designing program, Primer3 (http://www-genome.wi.mit.edu/cgi-bin/ primer/primer3.cgi) (Rozen and Skaletsky 2000); (3) set the primer optimum size, maximum size, and minimum size; (4) set the primer optimum Tm, maximum Tm, and minimum Tm; and (5) use one of the primers picked by the program.

\section{Optimization of PCR conditions}

DNA of four lines of common buckwheat (Kyusu, Canada, Miyazaki and Botansoba) was used to optimize the annealing temperature, PCR cycle and concentration of $\mathrm{MgCl}_{2}$ for 26 primers. Except these three variables all other PCR protocols were same as described below. PCR were run first at annealing temperature (Ta) of 62 with 40 cycles in a $1.5 \mathrm{mM} \mathrm{MgCl}_{2}$ concentration. Annealing temperature, PCR cycle number and $\mathrm{MgCl}_{2}$ were considered optimum if the single clear band was observed. If the band was not observed, annealing temperature was decreased by $2^{\circ} \mathrm{C}$. In the case of multiple bands, we increased the temperature by $2^{\circ} \mathrm{C}$. PCR cycle was increased if there was single but not clear band and cycle was decreased in the case of multiple but not clear bands. In some cases of very dim single band, concentration of $\mathrm{MgCl}_{2}$ was increased. If band intensity was low, we increased the cycle. If some bands were high intensity and some were low in multiple bands we decreased the cycle. We repeated this experiment until the single clear band of all 26 primers was achieved.

Table 1. Species and accessions of buckwheat used to check the PCR amplification using EST primers designed from common buckwheat

\begin{tabular}{llll}
\hline SN & Accession /line & Species & Remarks \\
\hline 1 & C2009 & Fagopyrum esculentum ssp ancestralis & Wild species \\
2 & C9740 & F. homotropicum $(2 \mathrm{x})$ & Wild species \\
3 & C0210 & F. homotropicum (4x) & Wild species \\
4 & C9722 & F. tataricum ssp tataricum & Cultivated species \\
5 & C9022 & F. tataricum ssp potanini & Wild species \\
6 & C8924 & F. cymosum (2x) & Wild species \\
7 & B9103 & F. cymosum (4x) & Wild species \\
8 & Hokkei 1 & F. tataricum ssp tatariucm & Cultivated species \\
9 & Hokkei 2 & F. tataricum ssp tatariucm & Cultivated species \\
10 & Hokuriku 4 & F. tataricum ssp tatariucm & Cultivated species \\
& & & \\
11 & Hokuriku 5 & F. tataricum ssp tatariucm & Cultivated species \\
12 & Ishisoba & F. tataricum ssp tatariucm & Cultivated species \\
13 & Tite 100 & F. tataricum ssp tatariucm & Cultivated species \\
14 & CV & F. tataricum ssp tatariucm & Cultivated species \\
15 & Kyusyu & F. esculentum ssp esculentum & Cultivated species \\
16 & Canada & F. esculentum ssp esculentum & Cultivated species \\
17 & Miyazaki & F. esculentum ssp esculentum & Cultivated species \\
18 & Botansoba & F. esculentum ssp esculentum & Cultivated species \\
\hline
\end{tabular}

\section{Primer selection and PCR run}

17 primers (Table 2) were selected after optimizing the PCR condition of 26 EST primers. Amplification reactions were performed in $0.5 \mathrm{ml}$ tubes in a volume of $30 \mathrm{ul}$ consisting of $14.7 \mu \mathrm{l}$ sterile distilled water, $6 \mu 15 \mathrm{x}$ buffer $(50 \mathrm{mM}$ Tris- $\mathrm{HCl} \mathrm{pH} 8.3,250 \mathrm{mM} \mathrm{KCl}, 500 \mu \mathrm{M}$ dNTP, $0.1 \%$ Triton X$100,0.05 \%$ Gelatin, $7.5 \mathrm{mM}$ ), $3 \mu \mathrm{l}$ of each primer of $2 \mu \mathrm{M}$ concentration, $0.3 \mu \mathrm{l}$ Taq DNA polymerase and $3 \mu \mathrm{l}$ template DNA. DNA amplification reaction were performed in a thermal cycler (GeneAmp PCR system 9700 and 2700 and TAKARA) using the following cycling program: one cycle of $94^{\circ} \mathrm{C}$ for $3 \mathrm{~min} ; 40$ cycles of $94^{\circ} \mathrm{C}$ for $0.30 \mathrm{~min}, 62^{\circ} \mathrm{C}$ (for primers $3,15,21,25,31,32,36,40,56,61,62,69,79$, 
87), $60{ }^{\circ} \mathrm{C}$ (for primers $23,35,64$ ) for $0.30 \mathrm{~min}, 72{ }^{\circ} \mathrm{C}$ for $0.30 \mathrm{~min}$; one cycle of $72{ }^{\circ} \mathrm{C}$ for $7 \mathrm{~min}$ followed by soaking in $4{ }^{\circ} \mathrm{C}$. PCR products were stored in fridge for further analysis.

\section{Electrophoresis run and data analysis}

PCR products were analyzed in 2\% Agarose S gel as described before. Based on band intensity, primers were related with accessions as showing high, medium and low bands. Number and percent of amplification of accessions by different primers were estimated.

Table 2. EST primers used in this experiment

\begin{tabular}{|c|c|c|c|c|c|}
\hline PN & Primer ID & Primer sequence & $\begin{array}{l}\text { Product } \\
\text { length, bp }\end{array}$ & $\mathrm{Tm}$ & $\mathrm{GC} \%$ \\
\hline \multirow[t]{2}{*}{3} & Fe_cb_0006_primer_4 & F: GAGGCTACGGATTTTCTGCC & 320 & 61.091 & 55.00 \\
\hline & & R: TCCTCGTCCTCTTCCTCCTC & & 60.870 & 60.00 \\
\hline \multirow[t]{2}{*}{15} & Fe_cb_0032_primer_0 & F: CCAAGCCCTTTTCTGCAACC & 192 & 64.126 & 55.00 \\
\hline & & R: CTTTTGCTTCCGTTTTCCCC & & 62.984 & 50.00 \\
\hline \multirow[t]{2}{*}{21} & Fe_cb_0045_primer_3 & F: CACATTCCTCAGATCCCACC & 314 & 60.326 & 55.00 \\
\hline & & R: TCTCСТCТСССТСТСССАТC & & 60.694 & 60.00 \\
\hline \multirow[t]{2}{*}{23} & Fe_cb_0050_primer_0 & F: TCAACTGCCAACTAACACCCA & 454 & 61.502 & 47.62 \\
\hline & & R: CACCAGCCCCACTCTCTTTC & & 62.145 & 60.00 \\
\hline \multirow[t]{2}{*}{25} & Fe_cb_0059_primer_0 & F: CAAGAGCGTTGTGTTACCGAG & 432 & 60.862 & 52.38 \\
\hline & & R: ACTGTGGGTCGGATTGTCAG & & 60.987 & 55.00 \\
\hline \multirow[t]{2}{*}{31} & Fe_cb_0073_primer_0 & F: CAAAGCACGTCCAAACAACA & 392 & 60.732 & 45.00 \\
\hline & & R: GGATAATGGCGGTGTCAAAA & & 60.701 & 45.00 \\
\hline \multirow[t]{2}{*}{32} & Fe_cb_0078_primer_0 & F: ACCGTTCCATGGGATCAAAG & 653 & 62.036 & 50.0 \\
\hline & & R: AGAACAACGCCATGCACAAA & & 62.599 & 45.0 \\
\hline \multirow[t]{2}{*}{35} & Fe_cb_0085_primer_0 & F: CAGCTAAGGCAGACGGTTGA & 148 & 61.499 & 55.00 \\
\hline & & R: TCATTGCCAGATCTCATTGGA & & 61.547 & 42.85 \\
\hline \multirow[t]{2}{*}{36} & Fe_cb_0087_primer_0 & F: TTТССССТTТСССТТСТТСС & 554 & 61.601 & 50.00 \\
\hline & & R: AAGACAGCCACCCAACTCAA & & 60.690 & 50.00 \\
\hline \multirow[t]{2}{*}{40} & Fe_cs_0003_primer_1 & F: AGCAGCAGCCATCCTTGTTC & 434 & 62.811 & 55.00 \\
\hline & & R: TCGCAAGGCTGTTGTTATCC & & 61.168 & 50.00 \\
\hline \multirow[t]{2}{*}{56} & Fe_cs_0174_primer_0 & F: CAAAGCAGGCAGAGAAAGCA & 559 & 61.753 & 50.00 \\
\hline & & R: GCAGATGCAAAATCAACTTCCC & & 63.032 & 45.45 \\
\hline \multirow[t]{2}{*}{61} & Fe_cs_0205_primer_0 & F: AGAGCAAGGAGGAGAATGGTG & 300 & 60.771 & 52.38 \\
\hline & & R: CTGAATCAAGGAGCGTTTGG & & 60.766 & 50.00 \\
\hline \multirow[t]{2}{*}{62} & Fe_cs_0206_primer_2 & F: GGGCAGACTCGGTGCTATTC & 291 & 62.086 & 60.00 \\
\hline & & R: GGTGAAGGGATTGTGGCTGT & & 62.279 & 55.00 \\
\hline \multirow[t]{2}{*}{64} & Fe_cs_0211_primer_0 & F: TCCCACGTTCATCGTTATAAACTC & 273 & 61.403 & 41.66 \\
\hline & & R: TGTTCATTTACGTCGGTTGTCC & & 62.038 & 45.45 \\
\hline \multirow[t]{2}{*}{69} & Fe_cs_0222_primer_0 & F: TGGCTGATAGCGGTGAAAGA & 377 & 61.848 & 50.00 \\
\hline & & R: GGATTGTGGGCTGACAAAGA & & 61.046 & 50.00 \\
\hline \multirow[t]{2}{*}{79} & Fe_cs_0247_primer_0 & F: CGAAACCACCAACAACTAAGCA & 429 & 62.290 & 45.45 \\
\hline & & R: CTAGGCGGTCGAGGATGAAG & & 62.187 & 60.00 \\
\hline \multirow[t]{2}{*}{87} & Fe_cs_0268_primer_1 & F: TTCACCATGAGTAGGTCGGG & 355 & 60.913 & 55.00 \\
\hline & & R: GATGGCAAGCAGTCAGGAAG & & 60.945 & 55.00 \\
\hline
\end{tabular}




\section{RESULTS AND DISCUSSION}

Primers with their amplification status are given in Table 3. Amplification products were different in band intensity (Table 4 and Figure 2). In most of the cases, the bands of $F$. homotropicum were with high intensity. All primers showed single band except in Accession C9022. Three primers 23, 31 and 69 produced very clear singe band (Table 5). Products ranged from 200 to $2000 \mathrm{bp}$. All primers amplified the genomic DNA of $F$. homotropicum (2x). 94.12\% of the total primers amplified the DNA of $F$. esculentum $\operatorname{ssp}$ ancestralis and $F$. esculentum ssp esculentum. DNA of $F$. cymosum (2x and $4 \mathrm{x})$ and $F$. tataricum ssp tataricum was amplified by least number of primers (58.82\%) (Figure 3). Eight primers amplified the DNA of all accessions.

The rate of successful amplification was found to be higher in more closely related species (Konishi 2006) eg F. esculentum ssp ancestralis (100\%) and diploid $F$. homotropicum $(79.6 \%)$ whereas it was lower in more distantly related species eg $F$. tataricum ssp potanini (25.9\%), diploid (36.7\%) and tetraploid $(33.3 \%) F$. cymosum. We had the similar results. Results indicated that the transferability of EST markers developed for common buckwheat decreased with an increase in genetic distance between them. It has been reported that the percentage of primer pairs that successfully amplified microsatellite loci was lower in polyploidy species as compared with their diploid counterparts. It is possible that the primer pairs which did not amplify EST loci in a given species under our conditions may become successful in amplifying EST loci in other species under differing annealing temperature.

The transferability of 207 Gossypium arboreum-derived expressed sequence tag-simple sequence repeat (EST-SSR) primer pairs was examined among 25 different diploid accessions representing 7 genomes and 23 Gossypium species (Guo et al 2006). They found that 124 of the $207(60 \%)$ primer pairs produced amplification products in all 25 accessions. The cross-species amplification of these ESTSSRs in 22 diploid species was $96.5 \%$.

The transferability with genomic SSR markers is generally low when cross-species analyses are conducted, while polymorphisms are numerous and some of the SSRs were found to be genome-specific (Guo et al 2006). Meanwhile EST-SSR markers derived from transcribed regions of the DNA produce a higher rate of transferability, but fewer polymorphisms. Based on wheat genomic SSR markers, the transferability from wheat to rye was found to be only $17 \%$ (Kuleung et al 2004); however, based on EST-SSR markers, the transferability from wheat to 18 Triticum-Aegilops species was found to be as high as $84 \%$ (Bandopadhyay et al 2004), and from tall fescue to seven grass species was found to be nearly 92\% (Saha et al 2004). Polymorphism frequency may be related to the mode of plant reproduction. It is low in self-pollinated species such as rice (43\%) and wheat (38\%), but is high in cross-pollinated species such as tall fescue and ryegrass (66\%) using the same markers.

The ability to transfer mapped STS markers between barley and wheat has been demonstrated (Taylor et al 2001). The transfer of mapped STS markers between cereals and forage grasses could provide PCRbased markers for comparative mapping in these species providing they amplify homologous sequences. Erpelding et al (1996) have shown that STS markers can be readily transferred between wheat and barley. A total of 21 primer sets derived from barley, T. tauchii and P. coerulescens loci have been assessed for their ability to amplify homologous sequences in L. perenne (Taylor et al 2001). Sequencing analysis of the PCR amplification products generated revealed that 11 primer sets (52\%) successfully amplified homologous fragments from 10 of the $18(55 \%)$ loci targeted. Analysis of homologous fragments generated in $L$. perenne reveals high levels of sequence identity and conservation in gene structure (ie presence, size and relative position of exons and introns) to barley, wheat and $P$. coerulescens homologues. The rate of success for primers designed from coding sequences was higher $(58 \%)$ than for primers derived from genomic DNA sequences (44\%). Primers that functioned well in one member of a genus are generally also successful in other members of that genus (Perry and Bousquet 1998). 
A high percentage of the common buckwheat-derived EST-PCR primer pairs resulted in successful amplification of DNA from other Fagopyrum species. EST-PCR fragments of Fagopyrum species other than common buckwheat can be compared their homologous to the original cDNA clones from common buckwheat. These markers should be useful for DNA fingerprinting, developing genetic linkage maps, and assessing genetic diversity within genus Fagopyrum. The markers which are homologous to the common buckwheat cDNA clones by DNA sequencing should also be useful for comparative mapping and possibly for examination of genetic relationships between species of the genus Fagopyrum.

The transferability among these different species presented here can increase the efficiency of transferring genetic information across species and further enhance their introgression into cultivated species by the molecular tagging of important genes existing in these species using the EST markers.

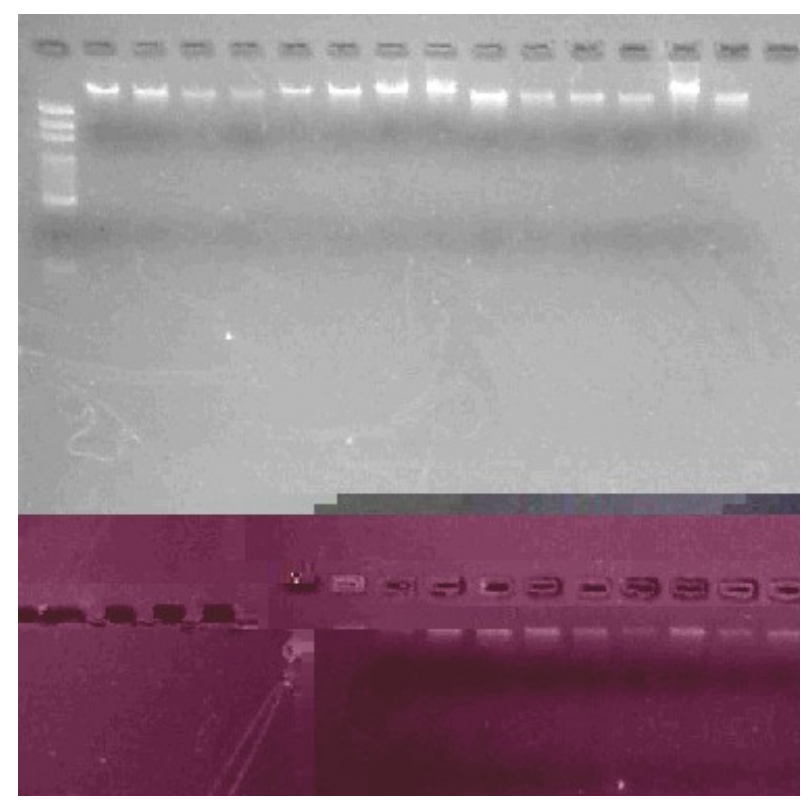

Table 3. Amplification condition of EST primers in different species

\begin{tabular}{|c|c|c|c|c|c|}
\hline $\mathrm{SN}$ & $\begin{array}{l}\text { Accession/ } \\
\text { line }\end{array}$ & Species & Primers amplified & $\begin{array}{l}\text { Primers } \\
\text { amplified, \% }\end{array}$ & $\begin{array}{l}\text { Non amplified } \\
\text { primers }\end{array}$ \\
\hline 1 & C2009 & $\begin{array}{l}\text { Fagopyrum esculentum ssp } \\
\text { ancestralis }\end{array}$ & $\begin{array}{l}3,15,21,23,25,31,32,35,40 \\
56,61,62,64,69,79,87\end{array}$ & $16(94.12)$ & 36 \\
\hline 2 & C9740 & F. homotropicum $(2 \mathrm{x})$ & $\begin{array}{l}3,15,21,23,25,31,32,35,36 \\
40,56,61,62,64,69,79,87\end{array}$ & $17(100)$ & - \\
\hline 3 & $\mathrm{C} 0210$ & F. homotropicum $(4 \mathrm{x})$ & $\begin{array}{l}3,15,21,23,32,31,35,40,56 \\
61,62,64,69,79,87\end{array}$ & $15(88.24)$ & 25,36 \\
\hline 4 & C9722 & F. tataricum ssp tataricum & $\begin{array}{l}3,15,21,23,32,31,35,61,62, \\
69,79\end{array}$ & $11(64.71)$ & $\begin{array}{l}25,32,36,40, \\
56,64\end{array}$ \\
\hline 5 & C9022 & F. tataricum ssp potanini & $\begin{array}{l}3,15,21,23,32,31,35,61,62, \\
69,79\end{array}$ & $11(64.71)$ & $\begin{array}{l}25,32,36,40, \\
56,64\end{array}$ \\
\hline 6 & C8924 & F. cymosum $(2 x)$ & $\begin{array}{l}3,15,21,23,32,31,35,61,69, \\
79\end{array}$ & $10(58.82)$ & $\begin{array}{l}25,32,36,40, \\
56,62,64\end{array}$ \\
\hline 7 & B9103 & F. cymosum $(4 \mathrm{x})$ & $\begin{array}{l}15,21,23,32,31,35,61,62, \\
69,79\end{array}$ & $10(58.82)$ & $\begin{array}{l}3,25,32,36 \\
40,56,64\end{array}$ \\
\hline 8 & Hokkei 1 & F. tataricum ssp tatariucm & $\begin{array}{l}3,15,21,23,32,31,35,61,62, \\
69,79\end{array}$ & $11(64.71)$ & $\begin{array}{l}25,32,36,40, \\
56,64\end{array}$ \\
\hline 9 & Hokkei 2 & F. tataricum ssp tatariucm & $\begin{array}{l}3,15,21,23,32,31,35,61,62, \\
69,79\end{array}$ & $11(64.71)$ & $\begin{array}{l}25,32,36,40, \\
56,64\end{array}$ \\
\hline 10 & Hokuriku 4 & F. tataricum ssp tatariucm & $\begin{array}{l}3,15,21,23,32,31,35,61,64, \\
69,79\end{array}$ & $11(64.71)$ & $\begin{array}{l}25,32,36,40, \\
56,62\end{array}$ \\
\hline
\end{tabular}




\begin{tabular}{|c|c|c|c|c|c|}
\hline $\mathrm{SN}$ & $\begin{array}{l}\text { Accession/ } \\
\text { line }\end{array}$ & Species & Primers amplified & $\begin{array}{l}\text { Primers } \\
\text { amplified, \% }\end{array}$ & $\begin{array}{l}\text { Non amplified } \\
\text { primers }\end{array}$ \\
\hline 11 & Hokuriku 5 & F. tataricum ssp tatariucm & $\begin{array}{l}3,15,21,23,32,31,35,61,62, \\
69,79\end{array}$ & $11(64.71)$ & $\begin{array}{l}25,32,36,40, \\
56,64\end{array}$ \\
\hline 12 & Ishisoba & F. tataricum ssp tatariucm & $\begin{array}{l}3,15,21,23,32,31,35,61,69, \\
79\end{array}$ & $10(58.82)$ & $\begin{array}{l}25,32,36,40, \\
56,62,64\end{array}$ \\
\hline 13 & Tite 100 & F. tataricum ssp tatariucm & $\begin{array}{l}3,15,21,23,32,31,35,61,62, \\
69,79\end{array}$ & $11(64.71)$ & $\begin{array}{l}25,32,36,40, \\
56,64\end{array}$ \\
\hline 14 & $\mathrm{CV}$ & F. tataricum ssp tatariucm & $\begin{array}{l}3,15,21,23,32,31,35,61,62, \\
69,79\end{array}$ & $11(64.71)$ & $\begin{array}{l}25,32,36,40, \\
56,64\end{array}$ \\
\hline 15 & Kyusyu & $\begin{array}{l}\text { F. esculentum ssp } \\
\text { esculentum }\end{array}$ & $\begin{array}{l}3,15,21,23,25,32,31,35,40, \\
56,61,64,69,79,87\end{array}$ & $16(94.12)$ & 36 \\
\hline 16 & Canada & $\begin{array}{l}\text { F. esculentum ssp } \\
\text { esculentum }\end{array}$ & $\begin{array}{l}3,15,21,23,25,32,31,35,40, \\
56,61,62,64,69,79\end{array}$ & $16(94.12)$ & 36 \\
\hline 17 & Miyazaki & $\begin{array}{l}\text { F. esculentum ssp } \\
\text { esculentum }\end{array}$ & $\begin{array}{l}3,15,21,23,25,32,31,35,40, \\
56,61,62,64,69,79,87\end{array}$ & $16(94.12)$ & 36 \\
\hline 18 & Botansoba & $\begin{array}{l}\text { F. esculentum ssp } \\
\text { esculentum }\end{array}$ & $\begin{array}{l}3,15,21,23,25,32,31,35,40, \\
56,61,62,64,69,79,87\end{array}$ & $16(94.12)$ & 36 \\
\hline
\end{tabular}

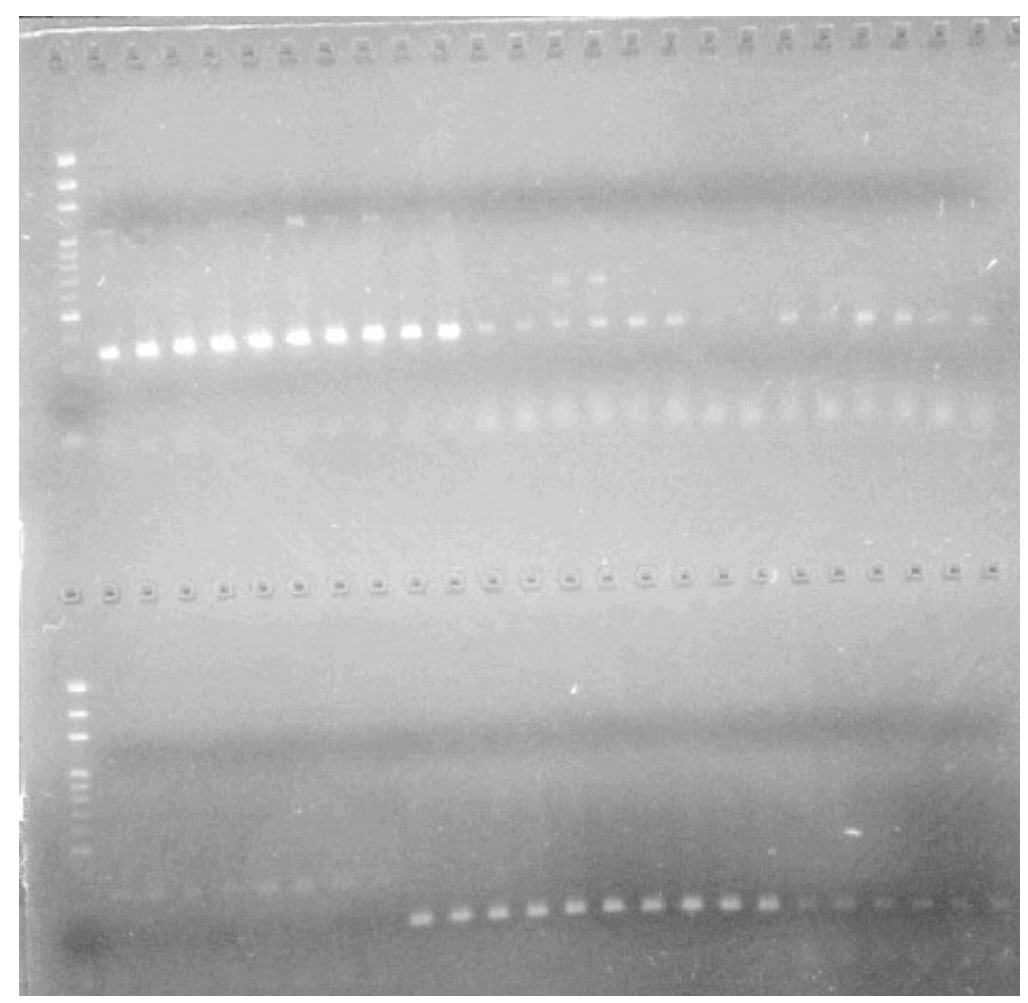

Figure 2. Amplification condition of primers 3 and 15 in different accessions and species of Fagopyrum (see Figure 1 for details). 
Table 4. Accessions based on the band intensity

\begin{tabular}{|c|c|c|c|c|}
\hline \multirow[t]{2}{*}{$\mathrm{SN}$} & \multirow[t]{2}{*}{$\mathrm{PN}$} & \multicolumn{3}{|c|}{ Bands intensity } \\
\hline & & High & Medium & Low \\
\hline 1. & 3 & $\begin{array}{l}\text { C2009, C9740, C0210, Kyusyu, Canada, } \\
\text { Miyazaki, Botansoba }\end{array}$ & - & $\begin{array}{l}\text { C9722, C9022, C8924, } \\
\text { Hokkei 1, Hokkei 2, Hokuriku } \\
\text { 4, Hokuriku 5, Ishisoba, Tite } \\
\text { 100, CV }\end{array}$ \\
\hline 2. & 15 & - & $\begin{array}{l}\text { C2009, C9740, C0210, B9103, } \\
\text { Hokke 1, Hokke 2, Hokuri Ku 4, } \\
\text { Hokuri Ku 5, Ishisoba, Tite 100, } \\
\text { CV, Kyusyu, Canada, Miyazaki, } \\
\text { Botansoba }\end{array}$ & C9722, C9022, C8924 \\
\hline 3. & 21 & $\begin{array}{l}\text { C2009, C9740, C0210, Kyusyu, Canada, } \\
\text { Miyazaki, Botansoba }\end{array}$ & - & $\begin{array}{l}\text { C9722, C9022, C8924, } \\
\text { Hokkei 1, Hokkei 2, Hokuriku } \\
\text { 4, Hokuriku 5, Ishisoba, Tite } \\
\text { 100, CV }\end{array}$ \\
\hline 4. & 23 & $\begin{array}{l}\text { C2009, C9740, C0210, B9103, Hokkei } \\
\text { 1, Hokkei 2, Hokuriku 4, Hokuriku 5, } \\
\text { Ishisoba, Tite 100, CV, Kyusyu, Canada, } \\
\text { Miyazaki, Botansoba }\end{array}$ & - & - \\
\hline 5. & 25 & - & - & $\begin{array}{l}\text { C2009, C9740, Kyusyu, } \\
\text { Canada, Miyazaki, Botansoba }\end{array}$ \\
\hline 6. & 31 & $\begin{array}{l}\text { C2009, C9740, C0210, C9722, C9022, } \\
\text { C8924, B9103, Hokkei 1, Hokkei 2, } \\
\text { Hokuriku 4, Hokuriku 5, Ishisoba, Tite } \\
\text { 100, CV, Kyusyu, Canada, Miyazaki, } \\
\text { Botansoba }\end{array}$ & - & - \\
\hline 7. & 32 & $\begin{array}{l}\text { C2009, C9740, C0210, Kyusyu, Canada, } \\
\text { Miyazaki, Botansoba }\end{array}$ & - & - \\
\hline 8. & 35 & - & - & $\begin{array}{l}\text { C2009, C9740, C0210, } \\
\text { C9722, C9022, C8924, } \\
\text { B9103, Hokkei 1, Hokkei 2, } \\
\text { Hokuriku 4, Hokuriku 5, } \\
\text { Ishisoba, Tite 100, CV, } \\
\text { Kyusyu, Canada, Miyazaki, } \\
\text { Botansoba }\end{array}$ \\
\hline 9. & 36 & - & - & C9740 \\
\hline 10. & 40 & C2009, C9740, C0210 & $\begin{array}{l}\text { Kyusyu, Canada, Miyazaki, } \\
\text { Botansoba }\end{array}$ & - \\
\hline 11. & 56 & - & C2009, C9740 & $\begin{array}{l}\text { C0210, Kyusyu, Canada, } \\
\text { Miyazaki, Botansoba }\end{array}$ \\
\hline 12. & 61 & $\begin{array}{l}\text { B9103, Hokkei 1, Hokkei 2, Hokuriku 4, } \\
\text { Hokuriku 5, Ishisoba, Tite 100, CV }\end{array}$ & $\begin{array}{l}\text { C2009, C9740, C0210, C9722, } \\
\text { C9022, C8924, Kyusyu, Canada, } \\
\text { Miyazaki, Botansoba }\end{array}$ & - \\
\hline 13. & 62 & - & $\begin{array}{l}\text { C2009, C9740, Kyusyu, Canada, } \\
\text { Miyazaki, Botansoba }\end{array}$ & $\begin{array}{l}\text { C0210, C9722, C9022, } \\
\text { B9103, Hokkei 1, Hokkei 2, } \\
\text { Hokuriku 5, Tite 100, CV }\end{array}$ \\
\hline 14. & 64 & - & $\begin{array}{l}\text { C2009, C0210, Kyusyu, Canada, } \\
\text { Miyazaki, Botansoba }\end{array}$ & - \\
\hline 15. & 69 & $\begin{array}{l}\text { C2009, C9740, C0210, C9722, C9022, } \\
\text { C8924, B9103, Hokkei 1, Hokkei 2, } \\
\text { Hokuriku 4, Hokuriku 5, Ishisoba, Tite } \\
\text { 100, CV, Kyusyu, Canada, Miyazaki, } \\
\text { Botansoba }\end{array}$ & - & - \\
\hline 16. & 79 & - & $\begin{array}{l}\text { B9103, Hokkei 1, Hokkei 2, } \\
\text { Hokuriku 4, Hokuriku 5, Ishisoba, } \\
\text { Tite 100, CV }\end{array}$ & $\begin{array}{l}\text { C2009, C9740, C0210, } \\
\text { C9722, C9022, C8924, } \\
\text { Kyusyu, Canada, Miyazaki, } \\
\text { Botansoba }\end{array}$ \\
\hline 17. & 87 & - & $\begin{array}{l}\text { C2009, C9740, C0210, Kyusyu, } \\
\text { Canada, Miyazaki, Botansoba }\end{array}$ & - \\
\hline
\end{tabular}

Number of band in all cases was single except in accession C9022 by primer 3. 
Table 5. Number and \% of accessions amplified by EST primers

\begin{tabular}{rrrrrrr}
\hline \multirow{2}{*}{ Primer } & \multicolumn{2}{l}{ Number of accessions in different bands intensity } & Product size, bp & Accessions amplified, \% \\
\cline { 2 - 5 } & High & Medium & Low & & \\
\hline 3 & 7 & - & 10 & 350,500 & 94.44 \\
\hline 15 & - & 15 & 3 & 200 & 100 \\
\hline 21 & 7 & - & 10 & 300 & 100 \\
\hline 23 & 15 & - & - & 400 & 100 \\
\hline 25 & - & - & 6 & 600 & 33.33 \\
\hline 31 & 18 & - & - & 800 & 100 \\
\hline 32 & 7 & - & - & 700 & 38.88 \\
\hline 35 & - & - & 18 & 200 & 100 \\
\hline 36 & - & - & 1 & 2000 & 5.55 \\
\hline 40 & 3 & 4 & - & 1200 & 38.88 \\
\hline & & - & & & 38.88 \\
\hline 56 & 8 & 2 & 5 & 600 & 100 \\
\hline 61 & - & 10 & - & 350 & 83.33 \\
\hline 62 & - & 6 & 9 & 350 & 33.33 \\
\hline 64 & 18 & 6 & - & 300 & 100 \\
\hline 69 & - & - & - & 400 & 100 \\
\hline 79 & - & 8 & 10 & 450 & 38.88 \\
\hline 87 & & 7 & - & 1400 & \\
\hline
\end{tabular}



Figure 3. Amplification condition of 17 EST primers in different species of Fagopyrum.

\section{ACKNOWLEDGEMENTS}

First author express sincere thanks to Dr MP Upadhyay and A Mudwari for their invaluable supports. This study was financially supported by JICA.

\section{REFERENCES}

Bandopadhyay R, S Sharma, S Rustig, R Singh, A Kumar, HS Balyan and PK Gupta. 2004. DNA polymorphism among 18 species of Triticum-Aegilops complex using EST-SSRs. Plant Sci. 166:349-356.

Echt CS, GG Vendramin, CD Nelson and P Marquardt. 1999. Microsatellite DNA as shared genetic markers among conifer species. Can. J. For. Res. 29:365-371.

Erpelding JE, NK Blake, TK Blake and LE Talbert. 1996. Transfer of sequence tagged site PCR markers between wheat and barley. Genome 39:802-810. 
Goff SA, D Ricke, TH Lan, G Presting, R Wang, M Dunn, J Glazebrook, A Sessions, P Oeller, H Varma, D Hadley, D Hutchison, C Martin, F Katagiri, BM Lange, T Moughamer, Y Xia, P Budworth, J Zhong, T Miguel, U Paszkowski, S Zhang, M Colbert, WL Sun, L Chen, B Cooper, S Park, TC Wood, L Mao, P Quail, R Wing, R Dean, Y Yu, A Zharkikh, R Shen, S Sahasrabudhe, A Thomas, R Cannings, A Gutin, D Pruss, J Reid, S Tavtigian, J Mitchell, G Eldredge, T Scholl, RM Miller, S Bhatnagar, N Adey, T Rubano, N Tusneem, R Robinson, J Feldhaus, T Macalma, A Oliphant and S Briggs. 2002. A draft sequence of the rice genome (Oryza sativa L. ssp. japonica). Science 296:92-100.

Guo W, W Wang, B Zhou and T Zhang. 2006. Cross-species transferability of G. arboreum -derived EST-SSRs in the diploid species of Gossypium. Theor. Appl. Genet. 112:1573-1581.

Harushima Y, M Yano, A Shomura, M Sato, T Shimano, Y Kuboki, T Yamamoto, SY Lin, BA Antonio, A Parco, H Kajiya, N Huang, K Yamamoto, Y Nagamura, N Kurata, GS Khush and T Sasaki. 1998. A high-density rice genetic linkage map with 2275 markers using a single F2 population. Genetics 148:479-494.

Konishi T, H Iwata, K Yashiro, U Tsumura, R Ohsawa, Y Yasui and O Ohnishi. 2006. Development and characterization of microsatellite markers for common buckwheat. Breeding Science 56:277-285.

Kuleung C, PS Baenziger and I Dweikat. 2004. Transferability of SSR markers among wheat, rye and triticale. Theor. Apple. Genet. 108:1147-1150.

Nakao Y, H Iwata, A Matsumoto, Y Tsumura and N Tomaru. 2001. Highly polymorphic microsatellite markers in Chamaecyparis obtuse. Can. J. For. Res. 31:2248-2251.

Perry DJ and J Bousquet. 1998. Sequence-tagged-site (STS) markers of arbitrary genes: The utility of black spruce-derived STS primers in other conifers. Theor. Appl. Genet. 97 :735-743.

Rowland LJ, AL Bhanaraj, JJ Polashock and R Arora. 2003. Utility of blueberry derived EST-PCR primers in related Ericaceae species. Hort. Science 38(7):1428-1432.

Rozen S and H Skaletsky. 2000. Primer3 on the WWW for general users and for biologist programmers. In: Methods in molecular biology: Bioinformatics methods and protocols (S Misener and SA Krawetz, eds). Totowa NJ, Vol. 132. Pp. 365-386.

Saha MC, MAR Mian, I Eujayl, JC Zwonitzer, LJ Wang and GD May. 2004. Tall fescue EST-SSR markers with transferability across several grass species. Theor. Apple. Genet. 109:783-791.

Sato K, H Tsujimoto and R von Bothmer. 2005. Introduction of genetic diversity into cereals from their wild relatives. Czech J. Genet. Plant Breed. 41 (Special Issue):103-107.

Taylor C, K Madsen, S Borg, MG Møller, B Boelt and PB Holm. 2001. The development of sequence-tagged sites (STSs) in Lolium perenne L.: the application of primer sets derived from other genera. Theor. Appl. Genet. 103:648-658.

Yu JK, M La Rota, RV Kantety and ME Sorrells. 2004. EST derived SSR markers for comparative mapping in wheat and rice. Mol. Gen. Genomics 271:742-751. 